\title{
Mobile recommender system based on smart city graph
}

\author{
Karwan Hoshyar Khalid Khoshnaw ${ }^{1}$, Zardasht Abdulaziz Abdulkarim Shwany ${ }^{2}$, \\ Twana Mustafa ${ }^{3}$, Shayda Khudhur Ismail ${ }^{4}$ \\ ${ }^{1}$ Department of Information and CommunicationTechnology, Erbil Techonology College, Erbil Polytechnic University, Erbil, Iraq \\ ${ }^{2}$ Department of Information Technology, Erbil Health and Medical Technical College, Erbil Polytechnic University, Erbil, Iraq \\ ${ }^{3}$ Department of Information Technology, Akre Technical College of Informatics, Duhok Polytechnic University, Duhok, Iraq \\ ${ }^{4}$ Network Department, Information and CommunicationTechnology Directory, Higher Education and Scientific Research, Erbil, Iraq
}

\begin{abstract}
Article Info
Article history:

Received Aug 11, 2021

Revised Dec 24, 2021

Accepted Jan 17, 2022

\section{Keywords:}

Graph models

Intelligent transportation

systems

Internet of things

ABSTRACT

Mobile recommender systems have changed the way people find items, purposes of intrigue, administrations, or even new companions. The innovation behind mobile recommender systems has developed to give client inclinations and social impacts. This paper introduces a first way to build a mobile recommendation system based on smart city graphs that appear topic features, user profiles, and impacts acquired from social connections. It exploits graph centrality measures to expand customized recommendations from the semantic information represented in the graph. The graph shows and chooses graph algorithms for computing chart centrality that is the center of the mobile recommender system are exhibited. Semantic ideas, for example, semantic transcendence and likeness measures, are adjusted to the graph model. Usage challenges confronted to settle execution issues are additionally examined.
\end{abstract}

Mobile recommender systems

Smart city
This is an open access article under the CC BY-SA license.

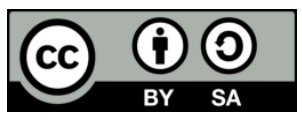

\section{Corresponding Author:}

Karwan Hoshyar Khalid Khoshnaw

Department of Information Technology, Erbil Technology College, Erbil Polytechnic University

Kerkuk Road, Erbil, Iraq

Email: karwan.khalid@epu.edu.iq

\section{INTRODUCTION}

A great deal of study in the field of intelligent-transport-systems, or intelligent transportation systems (ITSs), to alleviate mobility troubles, additionally by making use of unique methods details regarding the state of the road-way infrastructure [1], [2]. The diffuse availability of products and administrations through online and mobile applications makes it troublesome for end clients to choose the correct thing, as per their inclinations. A mobile recommender system must make utilization of various wellsprings of data for furnishing users with proposals of things that better relate to their desires. Those sources can incorporate user inclinations, thing depictions, or social data. A few methodologies have been proposed to handle the issue of selecting consequently the rundown of things that genuinely add to fulfill the necessities of end-users. Approaches in view of socioeconomics or displaying user profiles are situated to endeavor user components and inclinations to separate accessible decisions. The primary test of this approach is to make the user profile without any preparation. A few systems welcome users to choose their inclinations from a predefined rundown of classifications or permit the systems to extricate their profiles from different applications. Different systems enroll each user activity to fabricate models based upon the user's conduct powerfully. Some exploration works concentrate on gathering appraisals made by users that have assessed the item or administration, giving a direct view of the nature of the assessed thing. Different methodologies have concentrated on depicting the primary elements of each thing and attempting to match them with client necessities. 
Demonstrating intelligent city systems utilizing graphs has opened doors for investigating new choices for actualizing mobile recommender systems. Shrewd city, for example, influx centrality figured on graph-based designs provide interesting procedures to stand for the semantic transcendence of concepts consisting of users' dispositions as well as furthermore thing attributes. The first confirmation of the idea was refined and announced [3].

The rest of the paper is structured as follows: section 2 presents the mobile recommender system. After that, section 3 user modeling. Section 4 goes over picked graph design, semantic connections of the graph, centrality algorithm, and graph recommendation.

\section{MOBILE RECOMMENDER SYSTEMS}

One zone increasing of research is mobile recommender systems. With the expanding omnipresence of the web getting to smart-phones, it is presently conceivable to supply personalized, setting, touchy proposals. This is an especially troublesome territory of research as versatile information is more mindboggling than information that recommender systems frequently need to manage (it is heterogeneous, energetic, needs spatial as well as short-term auto-relationship, and has authorization and-also sweeping statement concerns [4]). Additionally, mobile recommender systems experience the impacts of the unwell effects of a transplant concern-proposals may not make a variance in all areas (as an example, it is incautious to recommend a formula in a zone where the greater majority of the fixings might not be obtainable) [5].

One situation of a mobile recommender system is one that provides conceivably useful driving programs for cab drivers in a city [6]. This system takes input details as global positioning system tips of the programs that cabbies took while working, which integrate area (range as well as longitude), time stamps, as well as operational condition (with or without travelers). It uses this information to prescribe a rundown of pickup concentrates along with a program, with the goal of advancing inhabitance times as well as benefits [7]. This type of system is plain area subservient, as well as since it has to work on a portable or implanted device, the estimation as well as vigor pre-requisites have to stay low [8], [9]. An additional situation of the versatile proposition is the thing that is generated for professional users. Utilizing GPS hints of the user and his plan, it recommends appropriate data relying upon his circumstance and interests as shown in the Figure 1.

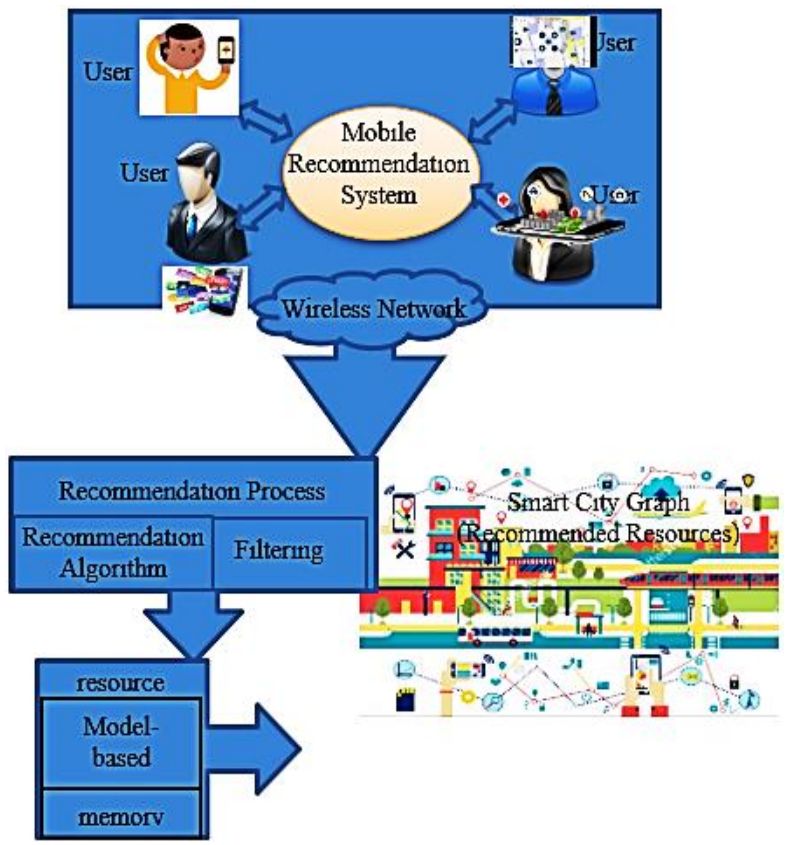

Figure 1. Mobile recommendation system framework based on smart city graph

The system utilizes machine learning methods and thinking forms keeping in mind the end goal to progressively adjust the mobile recommender system to develop the user's benefit. The author called his algorithm hybrid- $\varepsilon$-greedy [10]. Likewise, mobile recommendation systems have been effectively assembled utilizing the "Web of Data" as a hotspot for organized data. A decent case of such a system is smart museum 
[8]. The system utilizes semantic demonstrating, data recovery, and machine learning methods keeping in mind the end goal to suggest content coordinating user premiums, notwithstanding when given sparse or most minor user information.

\section{USER MODELING}

With a specific end goal to customize suggestions, it is necessary to recognize details concerning every user. Client designs are depictions of clients' requirements, goals, inclinations, interests, and practices alongside clients' statistic qualities [11]. A few user demonstrating approaches have been proposed, from the run of the mill weighted vectors to area ontologies. The creators characterized a user display in view of fuzzy logic and proposed a way to deal with induce the level of type nearness in a motion picture by abusing the labels allocated by the user [12]. The creators exhibited a straightforward credit esteem match lexicon to display the user clearly leads to the user's needs [13]. A wealthier user model is displayed, where the creators utilized a machine learning procedure to catch the client profile and to set it into an area cosmology [14].

\section{GRAPH MODEL}

Our mobile recommendation system depends on a graph depiction of users as well as objects of intrigue connected through ideas. Figure 1 demonstrates the chart shows where each hub falls in one of three classifications: user, term or object of intrigue and each edge speaks to the semantic connection between hubs: transcendence, similarity or friendship [15], [16]. Users are generally depicted by their tastes, inclinations, and intrigue (user model), while articles are portrayed by labels and catchphrases (object display). By doing this way, when a term is shared between a user as well as an object, it demonstrates the likelihood that the user could be occupied with that specific object. However, the object had never been seen or assessed by the user. A graph-based depiction permits us to apply graph algorithms (e.g., smart city) to find topological components, key connections, and vital (famous) hubs [17]. At that point, with these elements, we can make pertinent recommendations to users, for example, recommending companions or places [18]. Along these lines, the establishment of our recommender system depends on an information base constructed from both: a user show and an object display. Keeping in mind the end goal of developing the user and question models, we connected a phonetic investigation and protest content depictions.

Nevertheless, up to now extremely few investigations have been carried out on routing consisting of vehicle parking [19]. Park guidance and information (PGI) remedies are developed to increase the likelihood of finding a parking area [20], however without thinking about the opportunity to find a better remedy for the vehicle driver (e.g., a remedy that is not near the destination but less costly) [21], [22]. Furthermore, PGI remedies leave the burden of making the vehicle parking decision on the vehicle drivers by assessing all the available details in the destination area [23].

To fabricate the semantic relationships of the graph, it is important to obtain content depictions of users as well as articles. Accordingly, we have two accumulations: the user's content gathering (UTC) and the items content gathering (OTC), where every content portrayal is viewed as an archive D in a vector area display [24]. Three kinds of semantic relationships (sides of the graph): power, similitude, and fellowship. Each semantic connection joins diverse sorts of hubs. Power is the verge in between a user or a protest and a term, comparability is the verge in between 2 articles, and companionship is the verge between 2 users [25].

\subsection{Algorithms of centrality}

Centrality in graphs is usually utilized to quantify the importance of a pivot in a graph, particularly in SNA [26]. A mobile recommender system actualizes these centralities to determine the pertinence of individuals in the informal community. Some centrality measures like proximity and betweenness rely on the figuring of the briefest separation to attain every single other pivot in the graph. Algorithms to calculations to ascertain centralities are connected to the system of people so we can derive the most widespread pivot (degree), the limit of a pivot to attain whatever other in the system (closeness), as well as to differentiate the pioneers interconnected inside an area in the graph (betweenness) [27]. Degree centrality is a measure that checks the direct connections a pivot has, and in this manner, the pivot is in immediate contact. Nearness is defined as the reverse whole of the most limited ways to each other pivot, and betweenness is characterized as the quantity of briefest ways from all vertices to all others that go through that pivot [28], [29]. Centrality measures are figured over the system at a topological level given a sans range graph of people. In this way, these measures are not misusing our weighted chart, and they are connected just at an informal community level. Terms and protests of intrigue can be viewed as sub-diagrams of the worldwide system that can be abused by utilizing flow-based centrality measures. For a graph $G:=(V, E)$ with $\mathrm{n}$ vertices, the betweenness $C B(v)$ for vertex. 
In the Figure 2, user2 has the highest betweenness because it is between user1 and user3, who are between other entities. User1 and user3 have a slightly lower betweenness because they are essentialy only between their own cliques. Therefore, although user1 has a higher degree centrality, user2 has more importance in the network in certain respects.

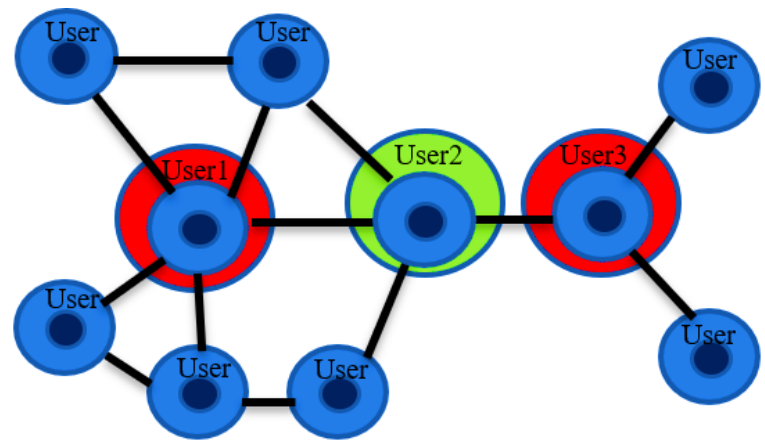

Figure 2. Mobile recommendation system framework based on smart city graph

\subsection{Graph recommendations}

As we have appeared, our recommender evince relies on the harmonious calculation of transcendence and similitude between things in the graph. As the graph develops from user collaborations between the user and objects of intrigue, the recommendations referrals obtain more exact after some time. In any case, with a specific end goal to give recommendations, calculation of centralities is required. We can suggest comparative things if the user is asking, what else?", then we can indicate him comparable things to the prescribed thing [30], [31]. Stream betweenness is utilized to suggest things the user can't miss" as a result of their pertinence in the system. Stream closeness is utilized to suggest focal things that could be things that the user would get a kick out of the chance to find", and unconventionality is utilized to show things to the user that are far from the more focal hubs in the system and could bring about a being different" impression [32].

\section{CONCLUSION}

We have exhibited a way to deal with a graph-based recommendation model that exploits shrewd, smart cities and recommends important to nationals and additionally guests in a smart city. The recommended demonstration communicates the semiotics of connections that subsist amongst clients as well as points of enthusiasm via terms that characterize a profile for the things. This new technique, utilizing especially circulation centralities, takes into consideration semantic transcendence of terms for characterizing and abusing the connections amongst client profile inclinations and also the illustrative qualities of purposes of intrigue.

Suggestions can then be extricated in view of the learning spoke to in the graph. So as to approve the recommendations model, the recommendation system was executed and has demonstrated that fascinating recommendations could be recommended to clients, considering their inclinations, as well as considering recommendations turning out from inclinations of different individuals from the interpersonal organization identified with them by the companionship relationship. The graph-based recommendation show additionally proposes to investigate purposes of premium that are altogether different to client inclinations, welcoming him to investigate new purposes of enthusiasm for the city.

\section{REFERENCES}

[1] S. Kwoczek, S. Di Martino, and W. Nejdl, "Predicting and visualizing traffic congestion in the presence of planned special events," Journal of Visual Languages \& Computing, vol. 25, no. 6, pp. 973-980, 2014, doi: 10.1016/j.jvlc.2014.10.028.

[2] S. Kwoczek, S. Di Martino, and W. Nejdl, "Stuck around the stadium, an approach to identify road segments affected by planned special events," in Intelligent Transportation Systems (ITSC), 2015 IEEE 18th International Conference on, IEEE, 2015, pp. 1255-1260, doi: 10.1109/ITSC.2015.206

[3] O. Cervantes, C. Thovex, and F. Trichet, "Dynamic recommendations for smart citizens based on socio-semantic network analysis," In: International Conference on Digital Intelligence, 2014.

[4] Y. Ge, H. Xiong, A. Tuzhilin, K. Xiao, M. Gruteser, and M. J. Pazzani, "An Energy-Efficient Mobile Recommender System," Proceedings of the 16th ACM SIGKDD Int'l Conf. on Knowledge Discovery and Data Mining, New York City, New York: ACM. 2011, pp. 899-908, doi: 10.1145/1835804.1835918. 
[5] L. Quijano-Sánchez, I. Cantador, M. E. Cortés-Cediel, and O. Gil, "Recommender systems for smart cities," Elsevier/Information Systems, vol. 92, p. 101545, 2020, doi: 10.1016/j.is.2020.101545.

[6] D. Bouneffouf, "Following the User's Interests in Mobile Context-Aware Recommender Systems The Hybrid-e-greedy Algorithm," Proceedings of the 2012 26th International Conference on Advanced Information Networking and Applications Workshops (PDF), Lecture Notes in Computer Science, IEEE Computer Society, 2012, pp. 657-662, doi: 10.1109/WAINA.2012.200.

[7] N. A. Othman, M. Z. N. Al-Dabagh, and I. AYDIN, "A New Embedded Surveillance System for Reducing COVID-19 Outbreak in Elderly Based on Deep Learning and IoT," 2020 International Conference on Data Analytics for Business and Industry: Way Towards a Sustainable Economy (ICDABI), 2020, pp. 1-6, doi: 10.1109/ICDABI51230.2020.9325651.

[8] T. Ruotsalo et al., "SMARTMUSEUM- A Mobile Recommender System for the Web of Data," Web Semantics, Science, Services and Agents on the World Wide Web. Elsevier, vol. 20, pp. 657-662, 2013, doi:10.1016/j.websem.2013.03.001.

[9] S. Schiaffino and A. Amandi, "Intelligent User Profiling In Bramer," Artificial Intelligence an International Perspective. LNCS, vol. 5640, pp. 193, 2009.

[10] N. A. Othman and I. Aydin, "A Smart School by Using an Embedded Deep Learning Approach for Preventing Fake Attendance," 2019 International Artificial Intelligence and Data Processing Symposium (IDAP), 2019, pp. 1-6, doi: 10.1109/IDAP.2019.8875883.

[11] S. R. Rizvi, S. Zehra, S. Olariu, and ASPIRE, "An agent-oriented smart parking recommendation system for smart cities," IEEE Intell. Transp. Syst. Mag., 2019, doi: 10.1109/MITS.2018.2876569.

[12] D. Anand and B. S. Mampilli, "Folksonomy-based fuzzy user profiling for improved recommendations," In Expert Systems with Applications, vol. 41, pp. 2424-2436, 2014, doi: 10.1016/j.eswa.2013.09.041.

[13] N. A. Othman, I. Aydin, and M. Karakose, "An Efficient Embedded Security System for Reduce Car Accident to Build Safer World Based On IoT," 2019 International Artificial Intelligence and Data Processing Symposium (IDAP), 2019, pp. 1-5, doi: 10.1109/IDAP.2019.8875933.

[14] M. Zanker and M. Jessenitschnig, "Case-studies on exploiting explicit customer requirements in recommender systems," User Model User-Adap Inter, Jour. Pers. Res., Springer, vol. 19, pp. 133-166, 2009, doi: 10.1007/s11257-008-9048-y.

[15] N. A. Othman and I. Aydin, "A face recognition method in the Internet of Things for security applications in smart homes and cities," 2018 6th International Istanbul Smart Grids and Cities Congress and Fair (ICSG), pp. 20-24, 2018, doi: 10.1109/SGCF.2018.8408934.

[16] Rothrock, V. Eyharabide, and A. Amandi, "Ontology-based user profile learning," Appl Intell, vol. 36, pp. 857-869, 2011, doi: 10.1007/s10489-011-0301-4

[17] E. H.-K. Wu, J. Sahoo, C.-Y. Liu, M.-H. Jin, and S.-H. Lin, "Agile urban parking recommendation service for intelligent vehicular guiding system," Intelligent Transportation Systems Magazine, IEEE, vol. 6, no. 1, pp. 35-49, 2014, doi: 10.1109/MITS.2013.2268549.

[18] F. Richter, S. Di Martino, and D. C. Mattfeld, "Temporal and spatial clustering for a parking prediction service, Tools with Artificial Intelligence (ICTAI)," IEEE 26th International Conference on, IEEE, 2014, pp. 278-282, doi: 10.1109/ICTAI.2014.49.

[19] C. Di Napoli, D. Di Nocera, and S. Rossi, "Agent negotiation for different needs in smart parking allocation," in Advances in Practical Applications of Heterogeneous Multi-Agent Systems, Springer International Publishing, 2014, pp. 98-109, doi: 10.1007/978-3-319-07551-8_9.

[20] F. Barile, C. Di Napoli, D. Di Nocera, and S. Rossi, "Evaluating the social benefit of a negotiation-based parking allocation," in Advances in Practical Applications of Agents, Multi-Agent Systems, and Sustainability: The PAAMS Collection, of Lecture Notes in Computer Science, Springer International Publishing, vol. 9086, pp. 15-26, 2015, doi: 10.1007/978-3-319-18944-4_2.

[21] Abidi, S. Krichen, E. Alba, and J. M. Molina, "A new heuristic for solving the parking assignment problem," Procedia Computer Science, vol. 60, pp. 312-321, 2015, doi: 10.1016/j.procs.2015.08.132.

[22] E. L. Merrer, G. Tredan, and Centralities, "Capturing the Fuzzy Notion of Importance in Social Graphs," in Proceedings of the Second ACM EuroSys Workshop on Social Network Systems, SNS '09. ACM, New York, NY, USA, pp. 33-38, 2009, doi: $10.1145 / 1578002.1578008$.

[23] M. Newman, "A measure of betweenness centrality based on random walks," Social Networks, vol. 27, no. 1, pp. 39-54, 2005, doi: 10.1016/j.socnet.2004.11.009.

[24] M. Karabatak, T. Mustafa, and C. Hamaali, "Remote Monitoring Real-Time Air pollution - IoT (Cloud Based)," 2020 8th International Symposium on Digital Forensics and Security (ISDFS), 2020, pp. 1-6, doi: 10.1109/ISDFS49300.2020.9116339.

[25] T. Mustafa and A. Varol, "Review of the Internet of Things for Healthcare Monitoring," 2020 8th International Symposium on Digital Forensics and Security (ISDFS), 2020, pp. 1-6, doi: 10.1109/ISDFS49300.2020.9116305.

[26] L. Quijano-Sánchez, I. Cantador, M.E. Cortés-Cediel, and O. Gil, "Recommender systems for smart cities," Information Systems vol. 92, p. 101545, 2020

[27] R. Wang et al., "TaxiRec: Recommending road clusters to taxi drivers using ranking-based extreme learning machines," IEEE Trans. Knowl. Data Eng., vol. 30, no. 3, pp. 585-598, 2018, doi: 10.1109/TKDE.2017.2772907.

[28] A. Hassani, P. D. Haghighi, S. Ling, P. P. Jayaraman, and A. Zaslavsky, "A smart car park recommender use case," Querying IoT services, in Proceedings of the 4th IEEE World Forum on Internet of Things, IEEE, 2018, pp. 619-624, doi: 10.1109/WFIoT.2018.8355158.

[29] T. Pinto, R. Faia, M. Navarro-Caceres, G. Santos, J. M. Corchado, and Z. Vale, "Multi-agent-based CBR recommender system for intelligent energy management in buildings," IEEE Syst. J., vol. 13, no. 1, pp. 1084-1095, 2019, doi: 10.1109/JSYST.2018.2876933.

[30] A. Agrawal, V. Raychoudhury, D. Saxena, and A. D. Kshemkalyani, "Efficient taxi and passenger searching in the smart city using distributed coordination," in Proceedings of the 21st International Conference on Intelligent Transportation Systems, IEEE, 2018, pp. 1920-1927, doi: 10.1109/ITSC.2018.8569728.

[31] I. Aydin and N. A. Othman, "A new IoT combined face detection of people by using computer vision for security application," 2017 International Artificial Intelligence and Data Processing Symposium (IDAP), 2017, pp. 1-6, doi: 10.1109/IDAP.2017.8090171.

[32] N. A. Othman and I. Aydin, "A new IoT combined body detection of people by using computer vision for security application," 2017 9th International Conference on Computational Intelligence and Communication Networks (CICN), 2017, pp. 108-112, doi: 10.1109/CICN.2017.8319366. 


\section{BIOGRAPHIES OF AUTHOR}

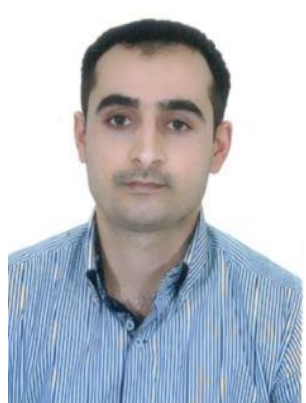

Karwan Hoshayr Khalid Khoshnaw (iD) 8d sc P He is received the Diploma degree in Mechanic from Erbil technology college, Erbil, Iraq. The B.Sc. degree in computer science from Cihan University, Erbil-Iraq. He is Registrar at Erbil technology college, Erbil Polytechnic University, Iraq. He Holds a M.Sc. degree in Computer Engineering from Firat University, Elazig-Turkey. He can be contacted at email: karwan.khalid@epu.edu.iq.

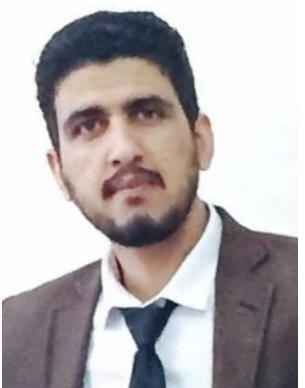

Zardasht Abdulaziz Adulkarim Shwany (iD) SC P He is received the B.Sc. degree in computer science from Salahaddin University, Erbil, Iraq. He is dairector of IT departent at Erbil Health and Medical Technical College, Erbil Polytechnic University, Erbil, Iraq. He Holds a M.Sc. degree in Computer Engineering from Firat University, Elazig-Turkey. He can be contacted at email: zardasht.abdulkarim@epu.edu.iq.

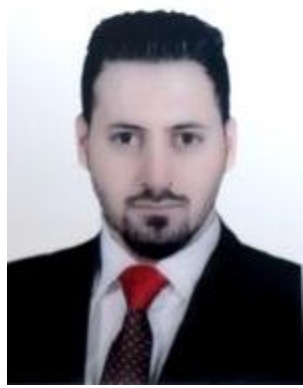

Twana Mustafa (D) SC $\mathrm{P}$ He is received the B.Sc. degree in computer science from Salahaddin University, Erbil, Iraq. He is Ass. Lecturer at IT departent at Erbil Noble technical Istitute, Erbil- Iraq. He Holds a M.Sc. degree in Computer Engineering from Firat University, Elazig-Turkey. September 2019- persistent Ph. D student at Firat zuniversity. He can be contacted at email: twana.mustafa@dpu.edu.krd.

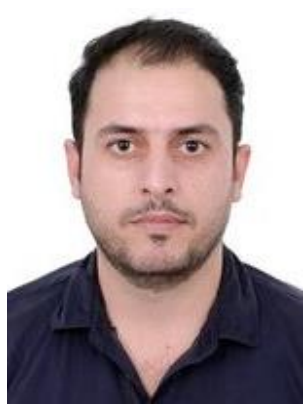

Shayda Kudhur Ismail (iD $8 \mathrm{SC}$ P $\mathrm{He}$ is received the Diploma degree in Computer from Erbil technology college, Erbil, Iraq. The B.Sc. degree in computer science from Salahaddin University, Erbil, Iraq. He is director of network department at Ministry of Higher Education and Scientific research, Erbil, Iraq. He Holds a M.Sc. degree in Computer Engineering from Firat University, Elazig-Turkey. He can be contacted at email: shayda.ismail@mhe-krg.org. 